\title{
Complications, disease profile and histological yield from percutaneous renal biopsy under real-time US guidance: A retrospective analysis
}

\author{
M Kruger, $M B C h B$ \\ E Loggenberg, MB ChB, MMed (Diag Rad) \\ Department of Diagnostic Radiology, Faculty of Health Sciences, University of the Free State, Bloemfontein
}

\begin{abstract}
Objective. The objective of the study was to evaluate (i) the technique used at Universitas Hospital in comparison with other international centres also performing renal biopsies, (ii) the disease profile in patients undergoing renal biopsies, (iii) the complications experienced during and/or after the procedure, and (iv) the histological yield of the biopsies (amount of nephrons per biopsy taken) using this technique.

Design. A retrospective descriptive analysis of all patients who underwent percutaneous renal biopsy under ultrasound (US) guidance at the Interventional Radiology Unit, Universitas Hospital, Bloemfontein, was undertaken for the period 1 January 2003 - 31 December 2008. Data obtained from the patients' files and histology reports were statistically analysed.

Results. A total of 112 patients qualified for inclusion in the study, all of whom had proof of renal failure and then had percutaneous renal biopsy performed under US guidance. The histology was diagnostic in 111 (99.1\%) of the cases, with more than 5 nephrons present in $105(93.5 \%)$ of the cases. Minor complications were found in 29 (25.8\%) of the patients, but no major complications were noted. Primary renal disease was found in $67(59.8 \%)$ of patients, and the renal pathology and failure in 45 (40.2\%) of the patients were shown histologically to be owing to systemic disease.

Conclusion. The technique utilised for performing percutaneous renal biopsy under US guidance at the Interventional Radiology Unit was shown to be safe, with a diagnostic histological yield comparable with international standards. A small majority or patients suffered primary renal disease in comparison with renal failure owing to systemic illness.
\end{abstract}

\section{Introduction}

Renal biopsies play a pivotal role in determining the diagnosis in patients with impaired renal function and with other renal diseases. The outcome of histological diagnosis after percutaneous renal biopsy can be utilised in not only making a diagnosis, but also planning treatment of the specific illness. ${ }^{1}$ The pathology and pathogenesis of glomerular disease have become far better understood since the introduction of ultrasound (US)-guided renal biopsies; this technique has transformed the diagnosis, treatment and long-term outcome of glomerular disease.
Biopsies are specifically necessary in cases of:

- unexplained renal failure

- nephrotic syndrome

- unexplained failure of a transplanted kidney

- progressively worsening renal function. ${ }^{2}$

Other indications for percutaneous renal biopsy include:

- suspected primary involvement of the kidneys by localised disease (such as with microscopic haematuria, unexplained macroscopic haematuria, proteinuria, impaired renal function and hypertension)

- renal involvement by systemic disease (including multiple myeloma, monoclonal gammopathy of uncertain significance, systemic lupus erythematosis, antiphospholipid syndrome, diabetes, systemic vasculitis and scleroderma)

- cardiac or liver transplantation with renal impairment (such as in long-term treatment with calcinereum inhibitors (e.g. cyclosporine) for autoimmune conditions). ${ }^{1}$

An absolute contraindication for this procedure is the inability of the patient to co-operate (e.g. when controlling breathing and lying in the prone position); children are therefore usually sedated for the biopsy. A horseshoe kidney is considered a major contraindication owing to the anomalous and unpredictable location of internal renal structures and because horseshoe kidneys often straddle the aorta.

Relative contraindications include:

- bleeding diathesis

- solitary kidney

- end-stage or near end-stage renal disease with bilaterally small kidneys

- although severe hypertension is universally listed as a contraindication to renal biopsy owingdue to risk of haemorrhage, no clinical studies are available to support this view. ${ }^{3}$

The difference in the diagnostic yield and complications of coaxial versus non-coaxial biopsy methods is a further consideration, but so far there is no evidence of any difference between the two methods in different studies. ${ }^{4}$

By international standards, over $99 \%$ of percutaneous renal biopsies are diagnostic. In a study of more than 1000 patients by Hergesell et al., only $1.2 \%$ of biopsy specimens contained no glomeruli or renal tissue; the median number of glomeruli per specimen was $9 .{ }^{5}$

Renal biopsy is a relatively safe procedure, with life-threatening complications occurring in less than $0.1 \%$ of biopsies. Standard care post-biopsy includes bedrest and close observation for 24 hours. Owing to the safety profile of the procedure, the international ongoing need 
for cost containment and the inherent safety of the procedure, renal biopsies are performed as outpatient procedures, with the patient being discharged after $6-8$ hours of observation. This applies to stable patients biopsied by experienced interventional radiologists or nephrologists. ${ }^{6}$

Stiles et al. found that where major complications occurred, these were detected in $46 \%$ of patients in $<4$ hours, in $79 \%$ of patients in $<8$ hours, and in $100 \%$ of patients in $\leq 12$ hours. They proposed that a 12 -hour observation period would therefore be sufficient in comparison with the 24-hour observation period previously recommended. ${ }^{3}$

Shina et al. showed that there was no difference in the rate of detection of complications after 6 hours instead of 24 hours in sedated or fully anaesthetised pediatric patients.

The relevance of anti-platelet agents, including aspirin, and whether these should be stopped pre-biopsy was addressed by Mackinnon et al.8 who found that minor complications were reduced when anti-platelet agents were stopped 5 days beforehand. These minor complications include macroscopic haematuria and perinephric haematoma that need no further intervention. Haemoglobin levels were also used to quantify the amount of blood loss, using a drop of more than $1 \mathrm{~g} / \mathrm{dl}$ without need for transfusion or intervention as a criterion. However, no difference was found in the rate of major complications. Major complications result in the need for intervention, such as a transfusion of blood products or more invasive procedures (radiological or surgical) as well as complications such as acute renal obstruction, renal failure, septicaemia or death. ${ }^{6,8}$

One of the most feared complications is the creation of arteriovenous fistulae (AVFs) or false aneurysms. Stiles et al. found AVFs in $10.8 \%$ of patients, by colour Doppler sonography. A significant disparity was shown in this complication between native kidney biopsies (4.4\%) and transplanted kidney biopsies (16.9\%). According to reports, 95\% of AVFs resolve spontaneously within 2 years, although haematuria, pain and urinary tract obstruction from blood clots had been reported, in rare cases up to 30 years after the biopsy. Routine screening for AVFs is, however, not recommended as clinically significant AVFs are uncommon. ${ }^{3}$ The cost implications and medico-legal aspects related to this procedure are also of specific interest in South Africa. ${ }^{5}$

\section{Methodology}

A retrospective descriptive analysis was conducted at the Interventional Radiology Unit, Universitas Hospital, Bloemfontein. Data were collected from 112 consecutive patients from 1 January 2003 - 31 December 2008. All patients included in the study had clinically relevant renal failure (indicated by elevated serum urea and creatinine levels and/or reduced 24 hour urinary creatinine-clearance). Other indicators for biopsy were proteinuria, haematuria or active urinary sediment (casts). Patients' ages ranged from 13 months to 72 years (average 41.5 years) and race was included in the analysis.

Information was collected from clinical notes, and histology results were obtained from hospital records. Data were captured on a data form and transferred to a Microsoft Excel spreadsheet for statistical analysis. Approval to conduct the investigation was granted by the Ethics Committee of the Faculty of Health Sciences, University of the Free State.

\section{Technique of US-guided percutaneous renal biopsy}

The biopsies were all performed in the interventional radiology theatre by a consultant radiologist with experience in performing vascular and interventional radiological procedures. A dedicated histologist was present at all biopsies to confirm that an adequate number of glomeruli were present in the biopsy specimen.

Children were given general anaesthesia, while adults had an infiltration of $10 \mathrm{ml}$ of $2 \%$ lignocaine as local anaesthetic. All patients were prone with a pillow under the abdomen to obliterate the normal lumbar lordosis. A second pillow was then placed under the chest to ensure adequate ventilation.

After sterile preparation of the patient and biopsy site, a Toshiba ECHO C US machine with a $3.75 \mathrm{MHz}$ curvilinear probe and biopsy guide for adult patients was used. A $3.75 \mathrm{MHz}$ narrow curvilinear (footprint) probe without a biopsy guide was used for paediatric patients. A sterile transparent plastic sleeve was used to cover the probe and cable. Standard adult biopsies were performed using a PROMAG ULTRA Automatic Biopsy Instrument with a throw (advancement) of $25 \mathrm{~mm}$. A $10 \mathrm{~cm}$-long 16-gauge PROMAG biopsy needle was used for paediatric renal biopsies and for transplant kidney biopsies. In adult native kidney renal biopsies, a 16-gauge, $16 \mathrm{~cm}$-long PROMAG biopsy needle was used.

\begin{tabular}{|lc|}
\hline & Table I. Patient gender $(\boldsymbol{N}=\mathbf{1 1 2})$ \\
\hline Male & $55(49.1 \%)$ \\
Female & $57(50.9 \%)$ \\
& \\
\hline
\end{tabular}

Table II. Patient age in years $(N=112)$

\begin{tabular}{rl}
\hline $0-10$ & $8(7.1 \%)$ \\
$11-20$ & $31(27.7 \%)$ \\
$21-30$ & $24(21.4 \%)$ \\
$31-40$ & $16(14.3 \%)$ \\
$41-50$ & $19(17.0 \%)$ \\
$51-60$ & $7(6.3 \%)$ \\
$61-70$ & $5(4.5 \%)$ \\
$>70$ & $2(1.8 \%)$
\end{tabular}

Table III. Complications $(N=112)$

\begin{tabular}{ll}
\hline None & $83(74.1 \%)$ \\
Microscopic haematuria & $27(24.1 \%)$ \\
Macroscopic haematuria & $2(1.8 \%)$ \\
& \\
\hline
\end{tabular}

Table IV. Glomeruli found in the histological specimen $(N=112)$

\begin{tabular}{ll}
\hline 0 & $1(0.9 \%)$ \\
$1-4$ & $6(5.4 \%)$ \\
$5-9$ & $48(42.8 \%)$ \\
$>9$ & $57(50.9 \%)$
\end{tabular}

Table V. Diagnosis after percutaneous renal biopsy $(N=112)$

Primary renal pathology

Systemic disease $67(59.8 \%)$ $45(40.2 \%)$ 
The lower pole of the left kidney was selected for biopsy, unless interpositioned bowel was present. A biopsy of the cortex was taken with the needle tip placed against the renal capsule before firing the biopsy instrument.

\section{Results}

Data were collected from the records of 112 patients during the study period. Their sex and age distribution are shown in Tables I and II.

No routine follow-up US was performed after biopsy. Urinary dipstick evaluation was performed prior to and after biopsy to exclude haematuria. Patients were monitored in the nephrology ward for 24 hours after the biopsy. The number of patients developing haematuria is shown in Table III.

No severe complications, including post-biopsy AVFs, need for blood transfusion, septicaemia or death were encountered.

Histological yield of glomeruli is shown in Table IV. Only 1 (representing $0.9 \%$ ) of the 112 patients who were biopsied had no glomeruli present. The number of glomuli per specimen exceeded 5 in 105 (93.5\%) patients.

The histological diagnosis, either as primary renal pathology or as renal disease as part of a systemic illness, is shown in Table V. Primary renal diseases include diseases such as nephrotic syndrome and glomerulonephritis. Systemic illness include systemic lupus erythematosis, diabetes and scleroderma.

\section{Discussion}

This retrospective descriptive analysis has the weakness that complications occurring after discharge might not have been captured, and the results might have underestimated the occurrence of minor complications. Furthermore, small perinephric haematomas would not have been documented as no routine US follow-up was done. Only haematuria would prompt post-biopsy US evaluation.

The analysis nonetheless provides data concerning the general safety of this procedure at our institution, as well as the diagnostic yield. Although the international trend is to discharge the patient after 12 hours in uncomplicated cases, ${ }^{3,6,7}$ the routine 24-hour inpatient followup is still used at our institution. With the pressures of cost containment within the public hospital system in Free State Province, one could argue that the possibility of discharging patients after 12 hours should be further investigated. Deficiencies in transport systems and large travelling distances raise concerns, however.

The technique employed was shown to be generally safe with a good diagnostic yield. The $0.9 \%$ of specimens that did not show glomeruli is in keeping with international results. ${ }^{4,5}$ The yield of $\geq 5$ glomeruli in the biopsy specimens in $93.5 \%$ of specimens also compares favourably. The non-coaxial method employed at our institution is diagnostic in over $99 \%$ of biopsies.

The direct, real-time evaluation of the renal tissue being sampled, as well the ability to verify correct needle positioning and depth, is important in obtaining a high percentage of diagnostic specimens. ${ }^{2-5}$ The size of the needle and correct patient positioning technique are also felt to be beneficial in the containment of complications and ensuring high diagnostic yields of glomeruli. The experience of the radiologist performing the biopsy is also important.

Renal biopsy in this setting is safe and has high diagnostic yield. The majority (59.8\%) of patients undergoing percutaneous renal biopsy suffered from primary renal disease.

1. Tompson CRV. Indications for renal biopsy in chronic kidney disease. Clinical Medicine 2003:3:513-516.

2. Alexoupoulos E. How important is renal biopsy in the management of patients with glomerular disease? Nephrol Dial Transplant 2001;16:83-85.

3. Stiles KP, Yaun CM, Chung EM, Lyon RD, Lane JD, Abbott KC. Renal biopsy in high-risk patients with medical diseases of the kidney. Am J Kidney Dis 2000;36:419-433.

4. Hatfield MK, Beres RA, Sane SS, Zaleski GX. Percutaneous imaging-guided solid organ core needle biopsy: Coaxial versus non-coaxial method. Am J Radiol 2008;190:413-417.

5. Hergesell O, Felten H, Andrassy K, Köhn K, Ritz E. Safety of ultrasound-guided percutaneous renal biopsy- retrospective analysis of 1090 consecutive cases. Nephrol Dial Transplant 1998:13:975-977.

6. Whittier WL, Korbet SM. Timing of complications in percutaneous renal biopsy. J Am Soc Nephrol 2004;15:142-147

7. Sinha MD, Lewis MA, Bradbury MG, Webb NJA. Percutaneous real-time ultrasound-guided renal biopsy by automated biopsy gun in children: Safety and complications. J Nephrol 2006;19:41-44.

8. Mackinnon B, Fraser E, Simpson, Fox JG, Geddes C. Is it necessary to stop anti-platelet agents before native renal biopsy? Nephrol Dial Transplant 2008;23(11):3566-3570. 